\title{
Nanoscale friction properties of graphene and graphene oxide
}

\author{
Diana Berman ${ }^{1}$, Ali Erdemir ${ }^{2}$, Alexander V. Zinovev ${ }^{3}$ and Anirudha V. Sumant ${ }^{*}$ \\ ${ }^{1}$ Center for Nanoscale Materials, 9700 S. Cass Ave, Argonne National Laboratory, \\ Argonne, IL, 60439 \\ ${ }^{2}$ Energy Systems Division, 9700 S. Cass Ave, Argonne National \\ Laboratory, Argonne, IL, 60439 \\ ${ }^{3}$ Materials Science Division, 9700 S. Cass Ave, Argonne National Laboratory, Argonne, \\ IL, 60439
}

\section{*Corresponding author e-mail: sumant@anl.gov}

\begin{abstract}
Achieving superlow friction and wear at the micro/nano-scales through the uses of solid and liquid lubricants may allow superior performance and long-lasting operations in a range of micromechanical system including micro-electro mechanical systems (MEMS). Previous studies have indicated that conventional solid lubricants such as highly ordered pyrolitic graphite (HOPG) can only afford low friction in humid environments at micro/macro scales; however, HOPG is not suitable for practical micro-scale applications. In this study, we explored the nano-scale frictional properties of multi-layered graphene films as a potential solid lubricant for such applications. Atomic force microscopy (AFM) measurements have revealed that for high-purity multilayered graphene (7-9 layers), the friction force is significantly lower than what can be achieved by the use of HOPG, regardless of the counterpart AFM tip material. We have demonstrated that the quality and purity of multilayered graphene plays an important role in reducing lateral forces, while oxidation of graphene results in dramatically increased friction values. Also, for the first time, we demonstrated the possibility of achieving ultralow friction for CVD grown single layer graphene on silicon dioxide. This confirms that the deposition process insures a stronger adhesion to substrate and hence enables superior tribological performance than the previously reported mechanical exfoliation processes.
\end{abstract}

\section{Introduction.}


As moving micro/nano mechanical systems are attracting a lot of industrial interests in many diverse applications, development of new solid lubricants that can reduce friction and wear and extend lifetime of the nano/micro devices becomes very important. Due to its unusual mechanical and tribological properties [1] graphene is one of such new candidates for lowering friction and extending the lifetime of devices.

At macroscale, in fact, the previous research has shown that just few-layers of graphene [2-3] can dramatically reduce wear (i.e., by as much as 4 orders of magnitude) and friction (by factors of 5 to 6), further confirming graphene's remarkable ability to combat friction and wear. Also, it was demonstrated that graphene works equally well in humid and dry environments [1], which is not possible for the commonly used solid lubricants including graphite.

Miniaturization of devices for practical applications calls for new lubricants that are effective at the nano- and micro-scale. In the case of graphene, the majority of the nano-scale computational and experimental studies demonstrated strong dependence of its tribological performance on the layer stacking and other structural peculiarities [4-11]. In particular, it was first suggested by simulations $[8,10]$ and later experimentally demonstrated [12-14] by the AFM lateral force measurements that friction of graphene decreases with the increasing number of layers due to less out-of-plane deformation in front of the scanning tip. Likewise, the ultralow friction of the HOPG (which is essentially made of well-ordered stack of multiple layers of graphene) has been reported under conditions of incommensurability at nano-scales [15], but at micro/macro scales, it has failed to afford super-low friction and wear especially under dry, inert, or vacuum test conditions.

Here, we demonstrate that chemical vapor deposition (CVD) grown multilayer graphene can exhibit ultra-low friction properties at nano-scale. It is important to note that such frictional behavior does not require any additional specific or restrictive environmental and operational conditions. Simply, the low friction can be achieved under ambient environment conditions at room temperature. Moreover, we show the novel way of controlling friction of graphene either by functionalization, such as oxidation of graphene layers, or by varying the adhesion between graphene and underlying silicon dioxide $\left(\mathrm{SiO}_{2}\right)$ surface. This opens up new possibilities for graphene as a potential solid lubricant for MEMS applications, where silicon is the most common platform material, but has very poor friction and wear properties. 


\section{Experimental procedure.}

To characterize the multi-layer graphene frictional behavior, the graphene layers were deposited using a thermal chemical vapor deposition (CVD) system on top of a $300 \mathrm{~nm}$ thick Nickel (Ni) thin film on $\mathrm{SiO}_{2}$ substrates. The graphene deposition was performed at $950{ }^{\circ} \mathrm{C}$ for 7 minutes followed by slow cooling of the system in argon environment to ensure the formation of a multilayer graphene film. Fig. 1 presents the scanning electron microscopy (SEM) images as well as Raman spectra of the samples used in the study. The number of layers was calculated from the Raman spectroscopy analysis performed with the He-Ne laser (wavelength: $633 \mathrm{~nm}$ ) . Based on the peak intensity ratio and FWHM (full width of half maximum) of 2D peak (at $\sim 2650$ $\mathrm{cm}^{-1}$ ), as well as the positions of $\mathrm{G}$ (at $\sim 1584 \mathrm{~cm}^{-1}$ ) and 2D peaks, the estimated thickness of resultant multi-layer graphene reaches $7-9$ layers $[16,17]$. It is important to notice that at such high thickness, the Raman signature starts to look similar to HOPG with the exception of smaller defect peak due to the misoriented nature of the stacked layers and several grain boundaries. 

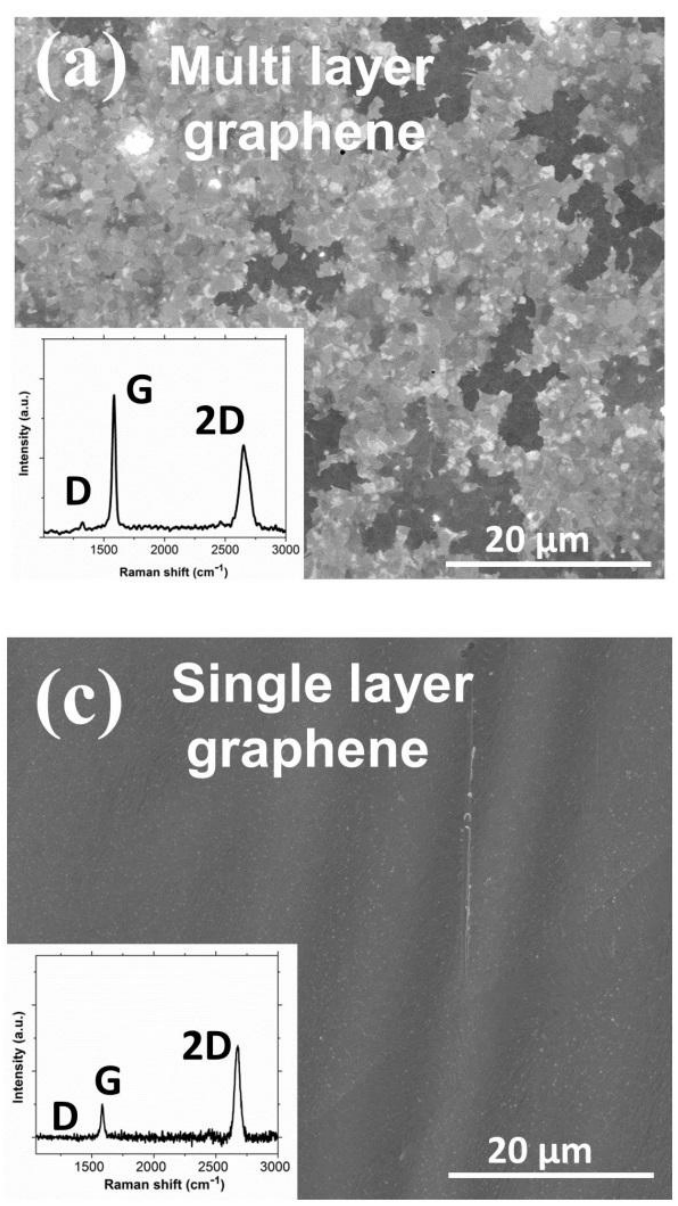
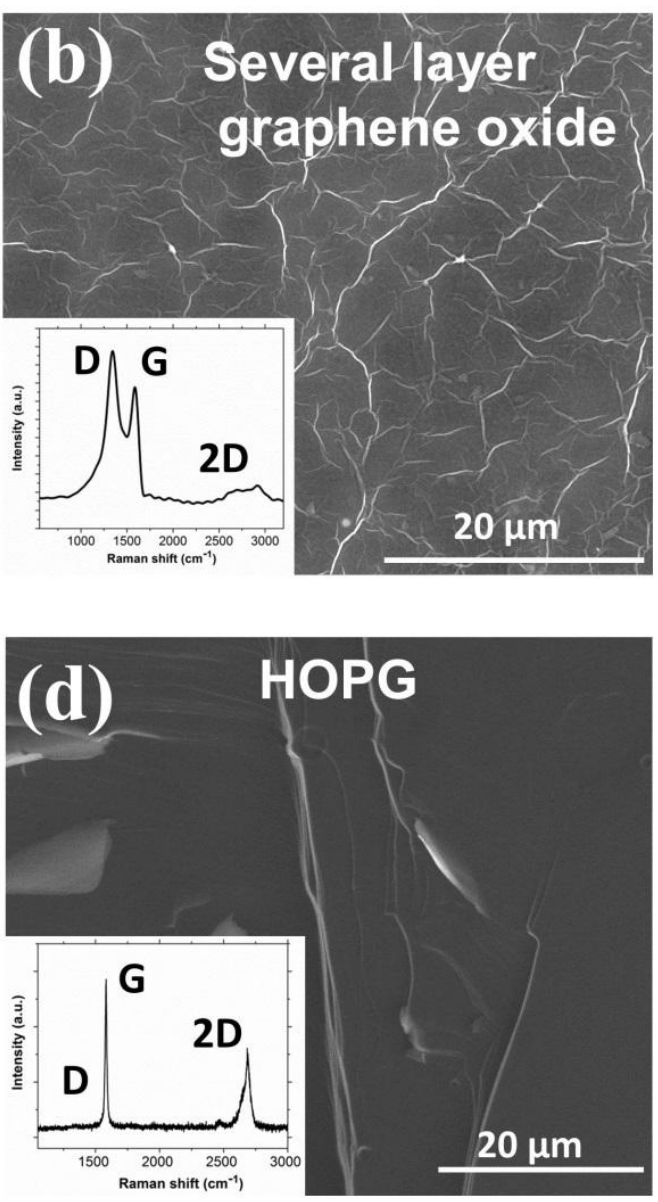

Figure 1 SEM images of the CVD-grown multilayer graphene on Nickel (a), solution processed multilayer graphene oxide (b), single layer CVD grown graphene on copper foil (c), and HOPG (d). Insets show the corresponding Raman signatures.

To prepare the graphene oxide samples (fig. 1b), we used commercially available water solution processed graphene oxide (SPGO) provided by Graphene Supermarket Inc. The concentration of graphene oxide in solution was $5 \mathrm{~g} / \mathrm{L}$ with $60 \%$ concentration of single layer graphene. The solution was applied on hydrophilic (processed in piranha solution) silicon dioxide $\left(\mathrm{SiO}_{2}\right)$ wafer $\left(1 \mathrm{ml}\right.$ of solution per $\left.1 \mathrm{~cm}^{2}\right)$ and evaporated in dry nitrogen environment, resulting in multilayer graphene oxide film on $\mathrm{SiO}_{2}$ wafer as shown in Fig. 1 b.

In case of single layer graphene (fig. 1c), the film was grown on copper foil using the widely established thermal CVD method [18,19]. Thickness of as-grown graphene was checked using Raman Spectroscopy. After deposition, the graphene layer is removed from one side of copper foil using oxygen plasma reactive ion etching (RIE) procedure. For achieving strong adhesion 
between single layer graphene and the underlying $\mathrm{SiO}_{2}$ substrate, a special and highly effective graphene transfer procedure was developed and implemented in our lab. Specifically, in order to provide a strong mechanical support to graphene during transfer process, a $\sim 300 \mathrm{~nm}$ thick poly(methyl methacrylate) (PMMA) protective layer was deposited on top of graphene/copper sample. After that, the $\mathrm{Cu}$ foil was etched with ammonium persulphate (0.1 M, Sigma Aldrich) and the PMMA/graphene film was rinsed in distilled water and lowered on $\mathrm{SiO}_{2}$ substrate and baked. The second PMMA layer, cured at room temperature was deposited on top to release the stress in the graphene layer and to provide the stronger adhesion between graphene and the underlying substrate. Then, the PMMA protective layer was removed using acetone to provide a single-layer graphene on steel flat sample. Special cleaning care was taken to eliminate any residual PMMA on the surface and the quality of resulting graphene films was confirmed by extensive Raman mapping of the surface. In our studies, two additional samples of high purity HOPG (fig. 1d) and $\mathrm{SiO}_{2}$ were used for the base line tests.

Atomic Force Microscope (AFM) has been used for the measurement of lateral friction forces of different carbon-based materials in the past [20,21] Here, we also use the lateral force measurement technique on a Veeco MultiMode 8 Scanning Probe Microscope at the contact mode for quantitative evaluation of graphene's tribological performance at nano-scale. Different AFM tip types with soft and hard cantilever material were utilized. The majority of the measurements were performed with the diamond like carbon (DLC) coated tip with force constant of $\mathrm{k} \sim 0.2 \mathrm{~N} / \mathrm{m}$ and silicon nitride ( $\mathrm{SiN}$ ) tip with $\mathrm{k} \sim 0.3 \mathrm{~N} / \mathrm{m}$ (provided by Ted Pella Inc.). In addition, a hard antimony doped silicon tip $(\mathrm{k} \sim 20 \mathrm{~N} / \mathrm{m})$ was used for friction response comparison. The lateral signal was recorded while ramping the normal load set point and the average friction force was determined by taking half of the difference between trace and retrace signals acquired during scanning. The resulting signal was then averaged over the scanning area and multiplied by a lateral force calibration factor, calculated using "wedge" method [22-24]. The height profile measurements were performed for the areas of $600 \mathrm{~nm}$ x $600 \mathrm{~nm}$ with the scanning speed of $1 \mathrm{~Hz}(0.6 \mu \mathrm{m} / \mathrm{s}$ scanning velocity), while lateral force data were acquired over $100 \mathrm{~nm} \times 100 \mathrm{~nm}$ scan size with $0.4 \mu \mathrm{m} / \mathrm{s}$ linear speed. The normal load axis is defined in a way, that zero value corresponds to zero externally applied load. The experimental uncertainties correspond to the standard deviation of the measured values. 
X-ray photoelectron spectroscopy (XPS) was performed by a home-built spectrometer with a hemispherical electron energy analyzer of $0.9 \mathrm{eV}$ energy resolution and a non-monochromated $\mathrm{Mg}$ K-alpha soft x-ray source source at $1253 \mathrm{eV}$.

\section{Results and discussion}

\subsection{Nanoscale friction on multilayer graphene and graphene oxide}

Comparative study of the frictional behavior of graphene and HOPG was performed with three different tip materials: DLC, SiN, and doped silicon. Fig. 2a shows the average friction response of $\mathrm{SiO}_{2}, \mathrm{HOPG}$, and CVD graphene (for each tip the friction is normalized to the value obtained for bare $\mathrm{SiO}_{2}$ sample) while the same load of $25 \mathrm{nN}$ is applied during the tests. Figs. $2 \mathrm{c}, 2 \mathrm{~d}$, and 2e present the detailed friction response, indicating the lowest friction values for high-purity CVD graphene. It should be mentioned that the applied normal load values are kept sufficiently low to prevent any plastic deformation of the tip. For the measurements on the graphite sample, the freshly cleaved surface has been immediately used, whereas, graphene was grown on the sample and transferred to the AFM system after some period of time. It is interesting to see that at nano-scale, both HOPG and CVD graphene show extremely low friction demonstrating their usefulness in developing low friction surfaces in MEMS and NEMS devices. 
(b)
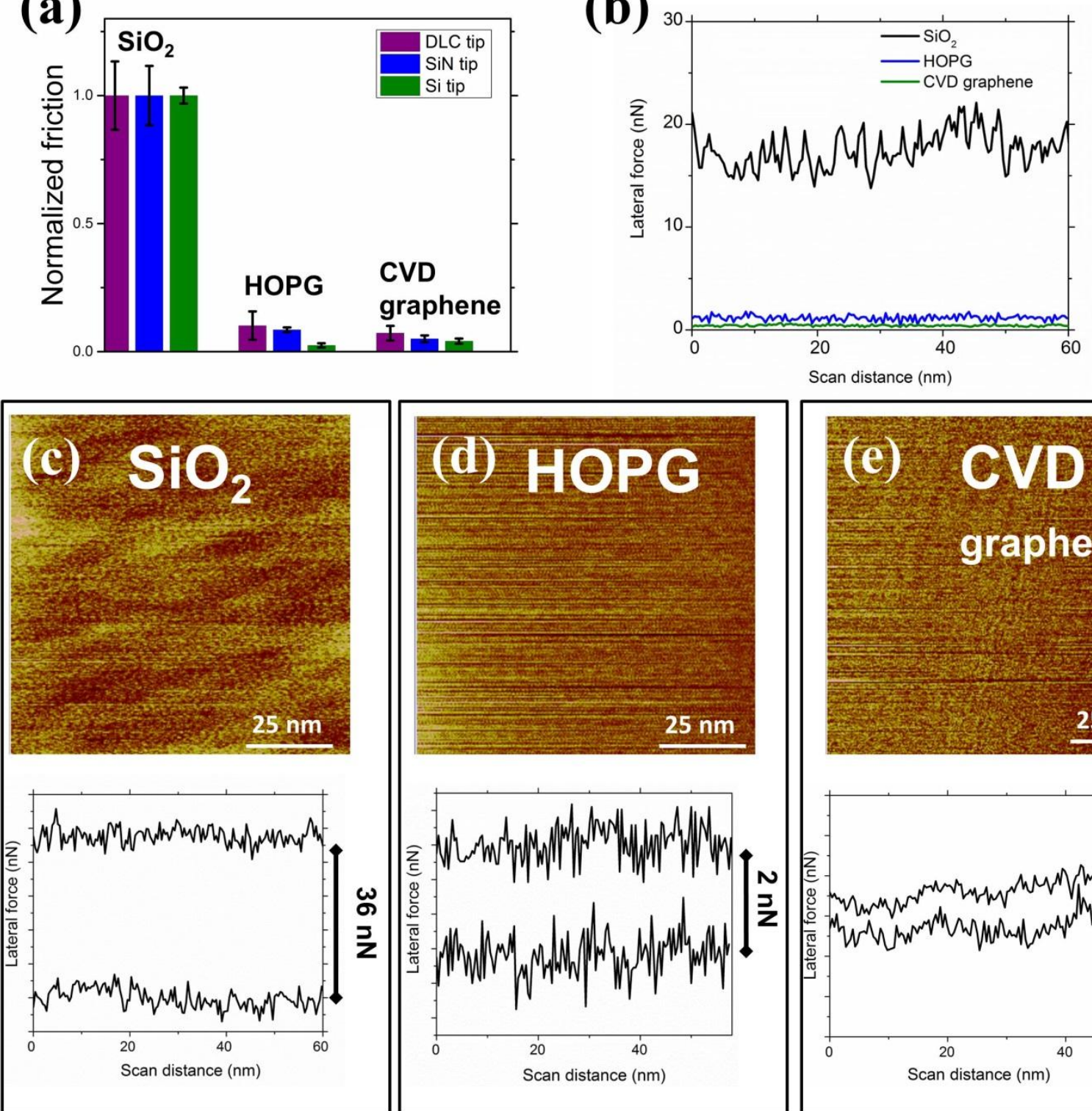

Scan distance $(\mathrm{nm})$

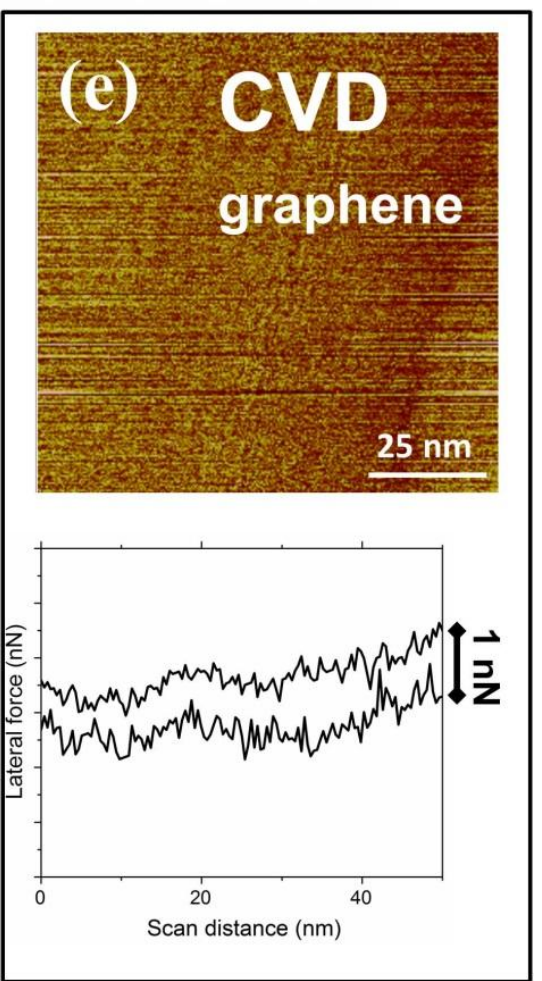

Figure 2 (a) Normalized friction response with (b) actual friction values for three different tips sliding on $\mathrm{SiO}_{2}, \mathrm{HOPG}$, and CVD grown graphene on Ni are presented. (c), (d) and (e) are the lateral force images and the detailed forward and backward lateral force signal for $\mathrm{SiO}_{2}, \mathrm{HOPG}$ and CVD-grown graphene respectively when the DLC coated tip was used. 25nN normal load was used for the measurements. 
Results in Fig. 2 indicate that CVD-grown multilayer graphene is capable of providing the friction values even lower than the ones for HOPG. Such a difference is attributed to the strong adhesion between graphene and the underlying nickel substrate, which essentially results in the reduced real contact area between the AFM tip and the sample due to the minimization of "puckering effect". In fact in previous microscale friction studies on multilayer CVD graphene on Ni such reduction in friction has been indeed observed [25] and the same mechanism seems to work in the present case at nanoscale.

The importance of this finding is that such multilayer graphene coatings may be used for MEMS devices through well-established CVD deposition and transfer procedure, while the HOPG is too thick or more difficult to implement for this purpose.

As demonstrated in fig.3, where the comparison between frictional behavior of multilayered graphene and graphene oxide is provided, the quality and functionalization of graphene films plays crucial role for its performance. Results indicate that for oxidized graphene the friction increases by a factor of more than 20. Using Density Functional Theory (DFT) calculations Wang et al. [26] predicted the atomic-scale friction of graphene oxide to be high. Moreover, in $[4,27]$ it was demonstrated that surface termination with oxygen highly affects the tribological performance of the materials. Therefore, we believe that the high oxidation ratio of graphene oxide results in larger formation of hydrogen bonds in the interlayer space due to presence of humidity and has higher binding energy which results in increased friction as observed. Also, the insertion of oxygen in graphene defects sites leads to $\mathrm{sp}^{3}$ bonds formation, thus demonstrating increase in the frictional response to the AFM tip scanning over the graphene oxide sheets.

XPS spectra (fig. 3e and 3f) are included to demonstrate the difference in the carbon composition for CVD grown graphene and for graphene oxide obtained from the commercial solution, as well as to highlight the oxidation level of graphene oxide. The composition analysis of graphene oxide (fig.3f) shows the corresponding atomic percentage carbon content to be $60 \%$, while oxygen to be $40 \%$. The high resolution C 1s spectra demonstrates chemical bonding of the oxygen to carbon as indicated by $\mathrm{C}-\mathrm{O}, \mathrm{C}=\mathrm{O}$ peaks at $286.8 \mathrm{eV}$ and $288.8 \mathrm{eV}$ respectively. 

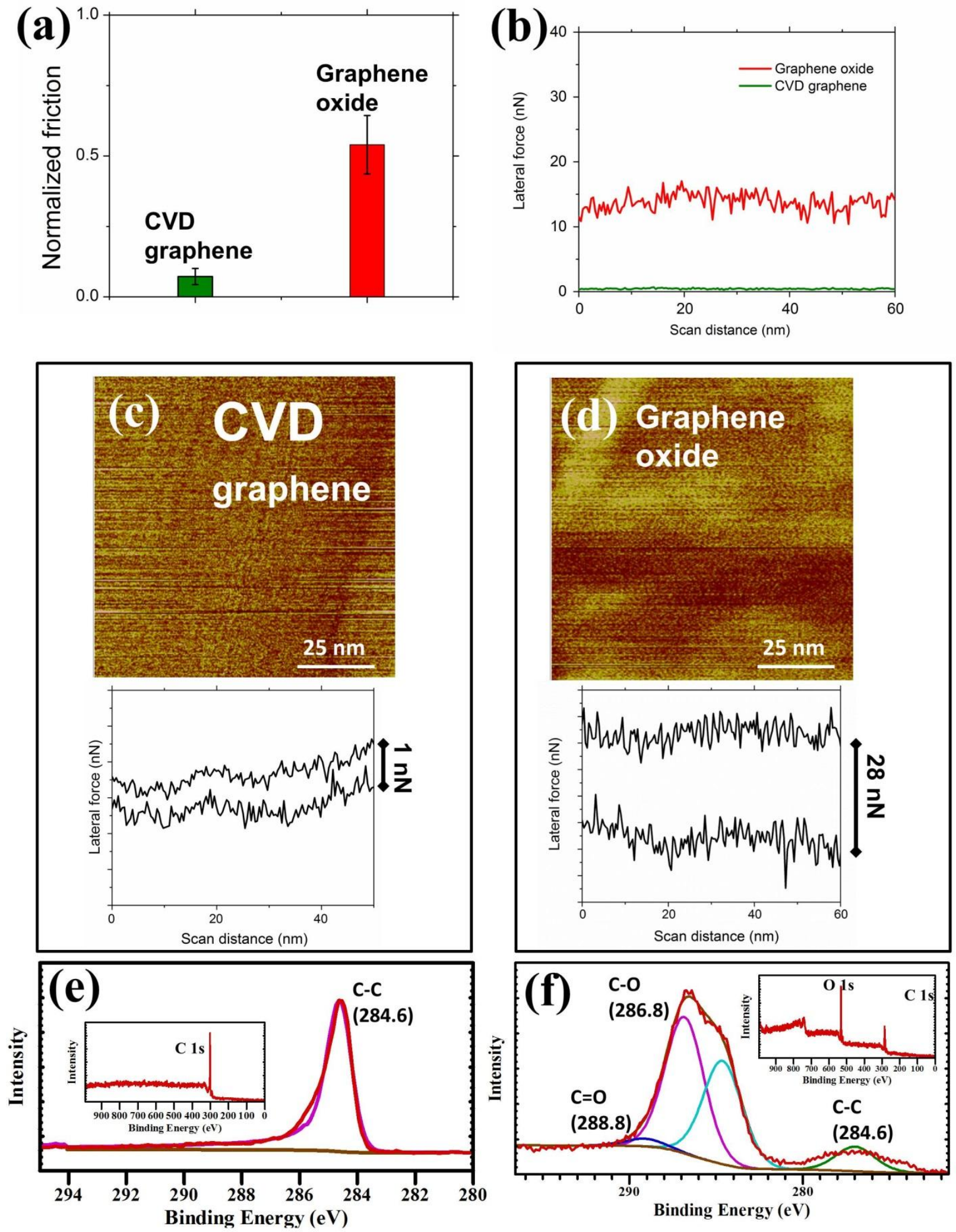


\subsection{Effect of interfacial adhesion of graphene to the substrate surface on nanoscale friction}

Here, we demonstrate the new insight on controlling the friction of single layer graphene by modifying the adhesion to the substrate through the different deposition methods. In the previous studies, the high friction values were observed on single layer graphene, mechanically exfoliated on $\mathrm{SiO}_{2}$ substrate $[13,28,29]$. Though, the importance of adhesion between graphene and the substrate was noted [4], there are no studies involving modified deposition methods to achieve low friction for single layer graphene on silicon dioxide.

On Fig. 4, using the modified PMMA transfer procedure, we demonstrate the graphene's ultralow friction regime at nano-scale for single layer graphene on $\mathrm{SiO}_{2}$ substrate sample. In particular, we show that weak adhesion to the substrate (regions represented as "bubbles" at the height profile) provides a noticeable increase in the friction response. Such increase is predictable from "puckering effect", when weak adhesion to the substrate enables the increase of the contact area in tip-graphene interaction. The adhesion effect on lowering the friction of graphene was demonstrated in case of graphene on muscovite mica substrate [4], The modified 
PMMA transfer method implemented in this paper resulted in strong adhesion of graphene with the underlying surface, in our case $\mathrm{SiO}_{2}$, but also may be extended to other materials. This is very important for using graphene in lubrication of Si MEMs devices and opens new possibilities for utilizing CVD graphene for MEMs lubrication.

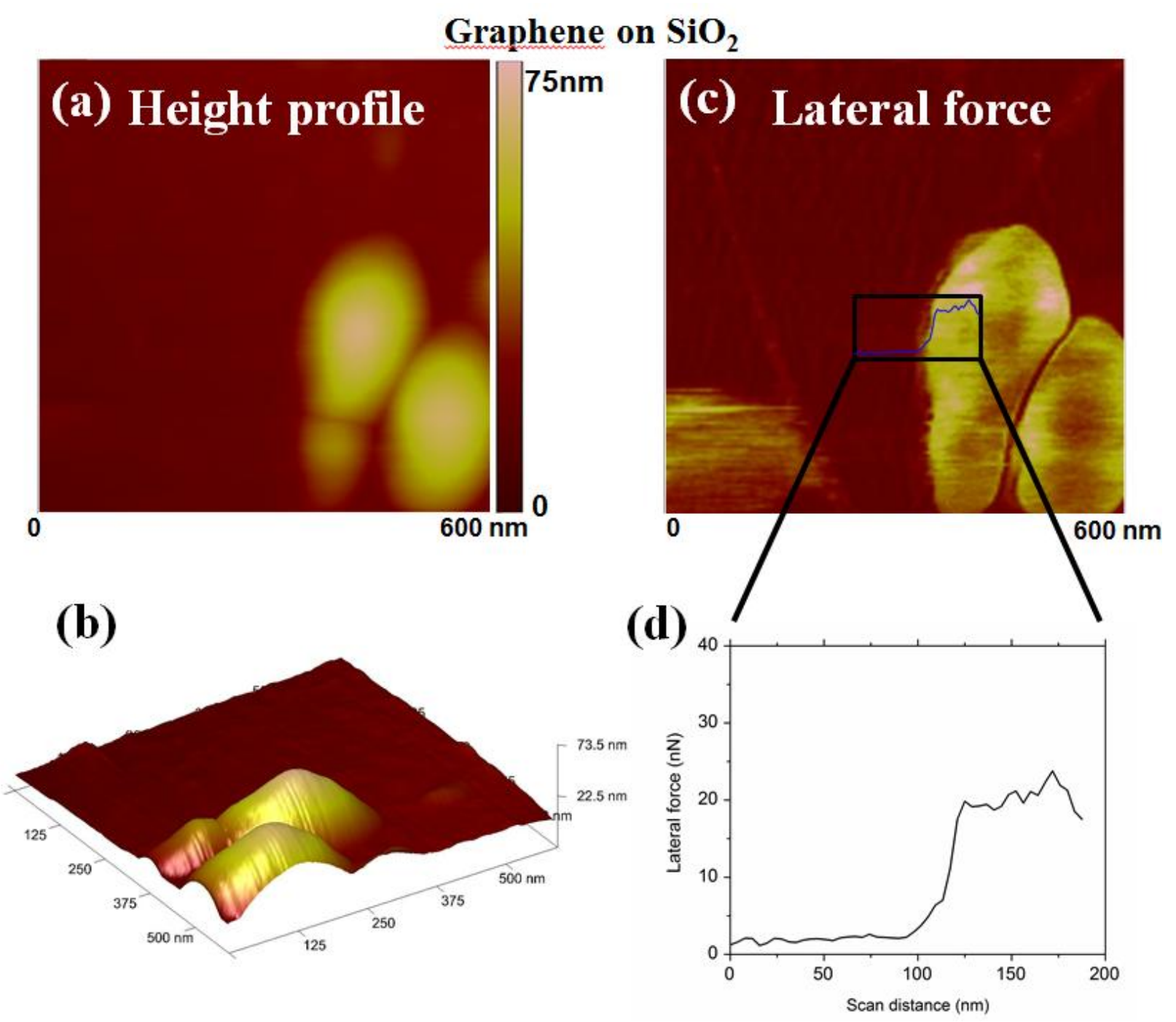

Figure 4. AFM height profile ( $a$ and $b$ ) and lateral force images (c) of single layer graphene transferred on $\mathrm{SiO}_{2}$ from copper foil. The image indicates the ultra-low friction of graphene in the regions of strong adhesion with the substrate while high friction occurs in the regions of weak adhesion (d). $25 \mathrm{nN}$ normal load is used for the measurements. DLC coated tip was used in the measurements. 


\section{Conclusion}

In the presented study we demonstrate the new ways of controlling the nanoscale friction response of graphene layers not only by varying the thickness, but also by changing the chemistry (oxidation) and changing the adhesion between graphene and underlying $\mathrm{SiO}_{2}$ substrate. In particular, the oxidation of multilayer graphene sheet results in increase in its friction response due to out-of plane nature of $\mathrm{sp}^{3}$ bonded carbon-oxygen and high binding energy of $\mathrm{O}-\mathrm{H}$ bonds formed in humid environment. Meanwhile, it is demonstrated that by modifying the method of graphene transfer process to $\mathrm{SiO}_{2}$ wafer and assuring the better adhesion of graphene with the substrate we can dramatically decrease the friction of even single layer graphene sheet. This opens new possibilities for graphene as a potential solid lubricant for MEMS applications, where silicon is the most common platform material but has very poor friction and wear properties.

\section{Acknowledgements}

Use of the Center for Nanoscale Materials was supported by the U. S. Department of Energy, Office of Science, Office of Basic Energy Sciences, under Contract No. DE-AC02-06CH11357. 


\section{References}

[1] D. Berman, A. Erdemir, A.V. Sumant, Graphene: a new emerging lubricant, Mater. Today 17 (2014) 31-42.

[2] D. Berman, A. Erdemir, A.V. Sumant, Few layer graphene to reduce wear and friction on sliding steel surfaces, Carbon 54 (2013) 454-459.

[3] D. Berman, A. Erdemir, A.V. Sumant, Reduced Wear and Friction Enabled by Graphene Layers on Sliding Steel Surfaces in Dry Nitrogen, Carbon 59 (2013) 167-175.

[4] Q. Li, C. Lee, R.w. Carpick, J. Hone, Substrate effect on thickness-dependent friction on graphene, Physica Status Solidi B 247 (2010) 2909-2914 .

[5] N. V. Medhekar, A. Ramasubramaniam, R. S. Ruoff, and V. B. Shenoy, Hydrogen bond networks in graphene oxide composite paper: structure and mechanical properties, ACS Nano 4 (2010) 2300.

[6] L. Xu, T.B. Ma , Y.Z. Hu, et al., Vanishing stick-slip friction in few-layer graphenes: the thickness effect, Nanotechnology 22 (2011) 285708.

[7] L. Xu, T.B. Ma , Y.Z. Hu, et al., Molecular dynamics simulation of the interlayer sliding behavior in few-layer graphene. Carbon 50, 1025-1032 (2012).

[8] P. Liu, Y.W. Zhang, A theoretical analysis of frictional and defect characteristics of graphene probed by a capped single-walled carbon nanotube, Carbon 49 (2011) 3687-3697.

[9] F. Bonelli, N. Manini, E. Cadelano, et al., Atomistic simulations of the sliding friction of graphene flakes. Eur. Phys. J. B, 70 (2009) 449-459, doi: 10.1140/epjb/e2009-00239-7.

[10] A. Smolyanitsky, J. P. Killgore, V. K. Tewary, Effect of elastic deformation on frictional properties of few-layer graphene, Phys. Rev. B 85 (2012) 035412.

[11] Y.F. Guo, W.L. Guo, C.F. Chen, Modifying atomic-scale friction between two graphene sheets: A molecular-force-field study. Phys. Rev. B 76 (2007) 155429, doi: 10.1103/PhysRevB.76.155429.

[12] Z. Deng, A. Smolyanitsky, Q. Li, X.Q. Feng, R.J. Cannara, Adhesion-dependent negative friction coefficient on chemically modified graphite at the nanoscale, Nature Materials 11 (2012) 1032-1037. 
[13] H. Lee, N. Lee, Y. Seo, et al. Comparison of frictional forces on graphene and graphite, Nanotechnology 20 (2009) 325701.

[14] L.Y. Lin, D.E. Kim, W.K. Kim, S.C. Jun, Friction and wear characteristics of multi-layer graphene films investigated by atomic force microscopy, Surf. and Coat. Technol. 205 (2011) 4868-4869.

[15] M. Dienwiebel, et al, Superlubricity of graphite, Phys. Rev. Lett 92 (2004) 126101.

[16] A. Das, B. Chakraborty et al, Raman spectroscopy of graphene on different substrates and influence of defects, Bull Mater Sci, 31, (2008) 579-584.

[17] Y. Hao, Y. Wang, L. Wang, Z. Ni, Z. Wang, R. Wang et al. Probing layer number and stacking order of few-layer graphene by Raman spectroscopy. Small 6 (2010) 195-200.

[18] R. Gulotty, S. Das, Y. Liu, A.V.Sumant, Effect of hydrogen flow during cooling phase to achieve uniform and repeatable growth of bilayer graphene on copper foils over large area. Carbon 77 (2014) 341-350.

[19] Li, X. S., Cai, W. W., Colombo, L. and Ruoff, R. S. Evolution of graphene growth on Ni and $\mathrm{Cu}$ by carbon isotope labeling. Nano Letters 9 (2009) 4268-4272.

[20] A.V. Sumant, D. S. Grierson, J. E. Gerbi, J. Birrell, U. D. Lanke, O. Auciello, J. A. Carlisle, and R. W. Carpick, Towards the ultimate tribological interface: Surface chemistry and nanotribology of ultrananocrystalline diamond, Adv. Mater. 17 (2005) 1039.

[21] R. J. Cannara, M. J. Brukman, K. Cimatu, A. V. Sumant, S. Baldelli, R. W. Carpick, "Nanoscale friction varied by isotopic shifting of surface vibrational frequencies," Science 318 (2007) 780.

[22] R.W. Carpick, The study of contact, adhesion and friction at the atomic scale by atomic force microscopy, Ph.D. Thesis, U. California, Berkeley (1997).

[23] Z. Deng, N.N.Klimov, S.D. Solares, T. Li, H. Xu, R. J. Cannara. Nanoscale interfacial friction and adhesion on supported versus suspended monolayer and multilayer graphene. Langmuir,29 (2013) 235-243.

[24] R.W. Carpick, D.F. Ogletree, M. Salmeron, A general equation for fitting contact area and friction vs load measurements. J Colloid and Interf Science 211 (1999) 395-400 
[25] K.-S. Kim, H.-J. Lee, C. Lee, S.-K. Lee, et al., Chemical vapor deposition-grown graphene: the thinnest solid lubricant. ACS Nano 5 (2011) 5107-5114.

[26] L-F. Wang, T-B. Ma, Y-Z Hu, H. Wang. Atomic-scale friction in graphene oxide: An interfacial interaction perspective from first-principles calculations. Phys Rev B 86 (2012) 125436.

[27] J. Chen, I. Ratera, J. Y. Park, and M. Salmeron, Velocity dependence of friction and hydrogen bonding effects, Phys. Rev. Lett. 96 (2006) 236102.

[28] X. Feng, S. Kwon, J. Y Park, et al. Superlubric Sliding of Graphene Nanoflakes on Graphene, ACS Nano 7 (2013) 1718-1724.

[29] C. Lee, Q. Li, W. Kalb, et al., Frictional Characteristics of atomically thin sheets, Science 328 (2010). 